International Journal of Bifurcation and Chaos, Vol. 10, No. 4 (2000) 797-810

(C) World Scientific Publishing Company

\title{
CHAOS SYNCHRONIZATION: A LAGRANGE PROGRAMMING NETWORK APPROACH
}

\author{
J. A. K. SUYKENS* and J. VANDEWALLE \\ Katholieke Universiteit Leuven, \\ Department of Electrical Engineering, \\ ESAT-SISTA, Kardinaal Mercierlaan 94, \\ B-3001 Leuven (Heverlee), Belgium
}

Received May 14, 1999; Revised August 30, 1999

\begin{abstract}
In this paper we interpret chaos synchronization schemes within the framework of Lagrange programming networks, which form a class of continuous-time optimization methods for solving constrained nonlinear optimization problems. From this study it follows that standard synchronization schemes can be regarded as a Lagrange programming network with soft constraining, where synchronization between state vectors is defined as a constraint to the dynamical systems. New schemes are proposed then which implement synchronization by hard and soft constraints within Lagrange programming networks. A version is derived which takes into account synchronization errors within the problem formulation. Furthermore Lagrange programming networks for achieving partial and generalized synchronization are given. The methods assume the existence of potential functions for the given systems. The proposed Lagrange programming networks with hard and soft constraining show improved performance on many simulation examples for identical and nonidentical chaotic systems. The schemes are illustrated on Chua's circuit, Lorenz attractor and $n$-scroll circuits.
\end{abstract}

\section{Introduction}

Chaos synchronization has been intensively studied in recent years [Chen \& Dong, 1998]. Schemes with mutual coupling and master-slave synchronization have been analyzed especially for ad hoc examples of general interest such as Chua's circuit and Lorenz attractor or for classes of nonlinear systems, e.g. Lur'e systems [Chen \& Dong, 1998; Wu \& Chua, 1994; Kapitaniak \& Chua, 1994; Kapitaniak et al., 1994; Kocarev et al., 1993; Suykens et al., 1997a, 1999]. Synchronization schemes have been investigated for identical and nonidentical chaotic systems, including aspects as local and global synchronization, robust synchronization, partial synchronization and generalized synchronization. Many of the synchronization methods have been applied to secure communications using chaos, where a message signal is defined as an external input of the scheme [Chen \& Dong, 1998; Wu \& Chua, 1994; Suykens et al., 1997c].

In this paper we make an interpretation of chaos synchronization schemes within the framework of Lagrange programming networks [Zhang \& Constantinides, 1992]. Being related to the Hopfield network [Hopfield, 1984] and other recurrent neural networks for nonlinear optimization [Kennedy \& Chua, 1988], Lagrange programming networks have been proposed as a class of continuous-time nonlinear optimization [Cichocki \& Unbehauen, 1994] for solving constrained nonlinear optimization problems with equality and/or inequality constraints [Fletcher, 1987]. Synchronization between

\footnotetext{
*Author for correspondence.

E-mail: johan.suykens@esat.kuleuven.ac.be
} 
state vectors of two nonlinear dynamical systems is defined then as a constraint to the dynamical system, assuming that there exists a potential function for the given systems, which is motivated by [Zubov, 1997] and potential function theory [Hazewinkel, 1991] with related results in circuit theory [Weiss et al., 1998].

As a result of the study we propose Lagrange programming networks for chaos synchronization that implement state vector synchronization by hard and soft constraints based upon an augmented Lagrangian. The synchronization constraint is realized in an asymptotical sense, which is different from the theory of differential-algebraic equations [Beardmore \& Song, 1998] where synchronization could be considered as an algebraic constraint. Certain standard synchronization schemes can be regarded as Lagrange programming networks with soft constraining only. New schemes are presented for full state synchronization, partial synchronization and generalized synchronization [Kocarev \& Parlitz, 1996]. The Lagrange programming networks for synchronization are applied to identical and nonidentical chaotic systems. Often synchronization is only achievable up to a small synchronization error, as shown in e.g. [Suykens et al., 1999] on robust synchronization of nonidentical Lur'e systems. A Lagrange programming network for chaos synchronization is proposed which takes into account synchronization errors in the problem formulation. In order to demonstrate the methods, Chua's circuit [Chua et al., 1986; Chua, 1994; Madan, 1993], Lorenz system [Lorenz, 1963] and $n$-scroll circuits [Suykens et al., 1997b; Yalcin et al., 2000] have been taken as simulation examples. In many cases the Lagrange programming network shows an improved performance compared to standard schemes that correspond to soft constraining. Theoretically speaking smooth versions to Chua's circuit and $n$-scroll circuits [Huang et al., 1996; Ponomarenko \& Matrosov, 1996] should be considered. However, simulation results suggest that the piecewise-linear nature of the circuits does not pose a problem for the investigated examples. At this point it is important to stress that no explicit construction of the underlying potential functions has to be made in order to simulate all of the synchronization schemes; only the existence of a potential function is needed, eventually in a generalized sense of potential function theory.
This paper is organized as follows. In Sec. 2 we discuss some standard synchronization schemes. In Sec. 3 Lagrange programming networks for constrained nonlinear optimization are shortly reviewed. In Sec. 4 Lagrange programming networks for chaos synchronization are proposed. The cases of partial and generalized synchronization are discussed in Sec. 5. Simulation examples are presented in Sec. 6.

\section{Synchronization Schemes}

In the context of synchronization of chaotic systems one studies schemes of the form [Chen \& Dong, 1998; Wu \& Chua, 1994]

$$
\left\{\begin{array}{l}
\dot{x}=f(x)+M(x-z) \\
\dot{z}=g(z)+N(x-z)
\end{array}\right.
$$

with $f(\cdot): \mathbb{R}^{n} \rightarrow \mathbb{R}^{n}, g(\cdot): \mathbb{R}^{n} \rightarrow \mathbb{R}^{n}$, state vectors $x, z \in \mathbb{R}^{n}$ and coupling matrices $M, N \in \mathbb{R}^{n \times n}$ which are often chosen diagonal. In the case of (1) the coupling between the two dynamical systems is mutual. For master-slave synchronization on the other hand one has the scheme

$$
\left\{\begin{aligned}
\mathcal{M}: \dot{x} & =f(x) \\
\mathcal{S}: \dot{z} & =g(z)+K(x-z)
\end{aligned}\right.
$$

with master system $\mathcal{M}$, slave system $\mathcal{S}$ and coupling matrix $K \in \mathbb{R}^{n \times n}$. Master-slave schemes are especially of interest in applications for secure communications using chaos, where the message signal is defined as an external input to the scheme $[\mathrm{Wu}$ \& Chua, 1994; Suykens et al., 1997].

Loosely speaking, for (1) and (2) synchronization means that $\|x-z\|_{2} \rightarrow 0$ as $t \rightarrow \infty$. Synchronization is often studied by stability analysis of the error system

$$
\dot{e}=f(x)-g(z)-K e
$$

for (2) where $e=x-z$ is the error signal. From the equations it is immediately clear that the analysis is simplified in the case of identical master and slave systems. For ad hoc examples such as Chua's circuit or Lorenz attractor synchronization has been studied in detail [Kocarev et al., 1993; Kapitaniak et al., 1994]. For the example of identical Lur'e systems a sufficient condition for global asymptotic stability of the error system may be derived from a quadratic 
Lyapunov function. The class of Lur'e systems includes e.g. Chua's circuit [Chua et al., 1986; Chua, 1994; Madan, 1993], $n$-scroll circuits [Suykens et al., 1997b] and coupled Chua's circuits [Kapitaniak \& Chua, 1994].

More specifically, for identical Lur'e systems one has the scheme

$$
\left\{\begin{array}{l}
\dot{x}=A x+B \sigma(C x)+M(x-z) \\
\dot{z}=A z+B \sigma(C z)+N(x-z)
\end{array}\right.
$$

with error system

$$
\dot{e}=(A+M-N) e+B \eta(e ; z)
$$

where $\eta(e ; z)=\sigma(C e+C z)-\sigma(C z)$ with $\sigma(\cdot)$ : $\mathbb{R}^{l} \rightarrow \mathbb{R}^{l}$ and $A \in \mathbb{R}^{n \times n}, B \in \mathbb{R}^{n \times l}, C \in \mathbb{R}^{l \times n}$. For $\eta$ and $\sigma$ one assumes a sector condition $[0, k]$. This means that for $\eta$ the inequality $\eta^{T} \Lambda[\eta-k C e] \leq 0$ holds $\forall e, z$ with $\Lambda$ a positive definite diagonal matrix. Employing a quadratic Lyapunov function $V(e)=e^{T} P e$ with $P=P^{T}>0$ it is straightforward to derive the following sufficient condition for global asymptotic stability of the error system:

$$
\begin{gathered}
{\left[\begin{array}{cc}
P(A+M-N)+(A+M-N)^{T} P & P B+k C^{T} \Lambda \\
B^{T} P+k \Lambda C & -2 \Lambda
\end{array}\right]} \\
<0
\end{gathered}
$$

where $<0$ denotes a negative definite matrix. In the master-slave scheme (2) synchronization may be regarded as a control problem where a feedback matrix $K$ has to be designed such that the slave is synchronized to the master system. The control is based upon the full state vectors $x, z$. In case there is a lack of full state information dynamic output feedback could be applied to the scheme [Suykens et al., 1997a]. Also observer-based synchronization design is relevant in this context [Morgül \& Solak, 1997].

For nonidentical Lur'e systems within the master-slave scheme

$$
\left\{\begin{aligned}
\mathcal{M}: \dot{x} & =A_{1} x+B_{1} \sigma\left(C_{1} x\right) \\
\mathcal{S}: \dot{z} & =A_{2} z+B_{2} \sigma\left(C_{2} z\right)+K(x-z),
\end{aligned}\right.
$$

with nonidentical matrices $A_{1}, A_{2}, B_{1}, B_{2}$ and $C_{1}$, $C_{2}$, conditions for robust synchronization have been derived in [Suykens et al., 1999]. Master and slave systems that behave in qualitative different ways with respect to each other (stable points, limit cycles versus chaos), can be synchronized up to a small synchronization error within (7).

In addition to synchronization of the type $\| x-$ $z \|_{2} \rightarrow 0$ as $t \rightarrow \infty$, one can also consider generalized synchronization [Kocarev \& Parlitz, 1996] which means that there exists a transformation $H$ : $\mathbb{R}^{n} \rightarrow \mathbb{R}^{m}$ such that a manifold $\{(x, z): z=H(x)\}$ is reached as $t \rightarrow \infty$ with a nonempty basin of attraction.

\section{Lagrange Programming Networks}

In this section we shortly review Lagrange programming networks as introduced by Zhang and Constantinides [1992]. Lagrange programming networks form a class of continuous-time optimization methods for solving constrained nonlinear programming problems with equality and/or inequality constraints. Here we review the equality constraint case.

Consider the optimization problem

$$
\min _{\xi \in \mathbb{R}^{r}} J(\xi) \quad \text { such that } h(\xi)=0
$$

with given functions $J(\cdot): \mathbb{R}^{p} \rightarrow \mathbb{R}$ and $h(\cdot): \mathbb{R}^{r} \rightarrow$ $\mathbb{R}^{s}$, where $r \leq s$. The functions $J$ and $h$ are assumed to be twice continuously differentiable. Based upon the Lagrangian

$$
\mathcal{L}(\xi, \lambda)=J(\xi)+\lambda^{T} h(\xi)
$$

where $\lambda \in \mathbb{R}^{r}$ denotes the vector of Lagrange multipliers, the Lagrange programming network for finding a local minimum to (8) is given by

$$
\left\{\begin{array}{l}
\dot{\xi}=-\frac{\partial \mathcal{L}(\xi, \lambda)}{\partial \xi} \\
\dot{\lambda}=\frac{\partial \mathcal{L}(\xi, \lambda)}{\partial \lambda}
\end{array}\right.
$$

which yields the equations

$$
\begin{cases}\dot{\xi}_{i}=-\frac{\partial J}{\partial \xi_{i}}-\sum_{j=1}^{s} \lambda_{j} \frac{\partial h_{j}}{\partial \xi_{i}}, & i=1,2, \ldots, r \\ \dot{\lambda}_{j}=h_{j}, & j=1,2, \ldots, s .\end{cases}
$$

For this network it can be shown that when $\left(\xi^{*}, \lambda^{*}\right)$ is a stationary point of $\mathcal{L}(\xi, \lambda)$ and the Hessian $\nabla^{2} \mathcal{L}\left(\xi^{*}, \lambda^{*}\right)$ is positive definite, then $\left(\xi^{*}, \lambda^{*}\right)$ is an asymptotically stable point of (11). Under the 
assumption that the Hessian $\nabla^{2} \mathcal{L}(\xi, \lambda)$ is positive definite everywhere, $V(\xi, \lambda)=1 / 2\|\partial \mathcal{L} / \partial \xi\|_{2}^{2}+$ $1 / 2\|h(\xi)\|_{2}^{2}$ is a Lyapunov function for the network.

The problem (8) can be convexified by considering the optimization problem

$$
\min _{\xi \in \mathbb{R}^{r}} J(\xi)+\frac{1}{2} \gamma\|h(\xi)\|_{2}^{2} \quad \text { such that } \quad h(\xi)=0
$$

with augmented Lagrangian

$$
\mathcal{L}_{\gamma}(\xi, \lambda)=J(\xi)+\lambda^{T} h(\xi)+\frac{1}{2} \gamma\|h(\xi)\|_{2}^{2} .
$$

In this case the local convexity assumption $\nabla^{2} \mathcal{L}\left(\xi^{*}, \lambda^{*}\right)>0$ is weakened. This augmented Lagrangian yields the Lagrange programming network

$$
\left\{\begin{array}{r}
\dot{\xi}_{i}=-\frac{\partial J}{\partial \xi_{i}}-\sum_{j=1}^{s} \gamma h_{j} \frac{\partial h_{j}}{\partial \xi_{i}}-\sum_{j=1}^{s} \lambda_{j} \frac{\partial h_{j}}{\partial \xi_{i}} \\
i=1,2, \ldots, r \\
\dot{\lambda}_{j}=h_{j}, \quad j=1,2, \ldots, s .
\end{array}\right.
$$

This network makes use of hard and soft constraints in order to solve the optimization problem, while in (11) only hard constraints are implemented.

\section{Chaos Synchronization and Lagrange Programming Networks}

Now, let us establish the link between the previous two sections and interpret the chaos synchronization schemes with respect to Lagrange programming networks.

For a system

$$
\dot{x}=f(x)
$$

with right-hand side defined in an $n$-dimensional Euclidean space and $f$ being a real-valued, continuous and continuously differentiable function in $x$, Poincaré and Lyapunov have shown that (15) can be transformed to the form

$$
\dot{x}=\frac{\partial \varphi(x)}{\partial x}
$$

by introducing new independent variables [Zubov, 1997] (potential systems of differential equations pp. 298-301). Consider again the vector fields $f(x)$, $g(z)$ as in (1). In the sequel we have to assume then the existence of potential functions $U_{1}(x)$ and $U_{2}(z)$ for the given vector fields $f(x), g(z)$ such that

$$
\begin{aligned}
& f(x)=-\frac{\partial U_{1}(x)}{\partial x} \\
& g(z)=-\frac{\partial U_{2}(z)}{\partial z}
\end{aligned}
$$

without the need actually for constructing $U_{1}(x)$, $U_{2}(z)$. This assumption is reasonable for a large class of nonlinear systems being further motivated in the area of circuit theory by the work on Brayton-Moser mixed potential functions [Weiss et al., 1998] and potential function theory [Hazewinkel et al., 1991]. According to [Hazewinkel et al., 1991] the test for the fields to be the gradient of a potential is that their Jacobian matrix is symmetric, which indicates that the field is rotation free. However, in the sequel we will assume the existence of a potential function also in the case of nonsymmetric Jacobian matrices, thereby relying on [Zubov, 1997] and further possible generalizations of potential functions [Hazewinkel et al., 1991]. The latter are characterized then in another way than (17) and would require a different notation than (17) in the further derivations.

Let us consider then the optimization problem

$$
\min _{x, z \in \mathbb{R}^{n}} J(x, z)=U_{1}(x)+U_{2}(z)+\frac{1}{2} \gamma\|x-z\|_{2}^{2}
$$

such that

$$
x-z=0
$$

with augmented Lagrangian

$$
\begin{aligned}
\mathcal{L}_{\gamma}(x, z, \lambda)= & U_{1}(x)+U_{2}(z) \\
& +\frac{1}{2} \gamma\|x-z\|_{2}^{2}+\lambda^{T}(x-z)
\end{aligned}
$$

where $\gamma$ is a positive real constant. The synchronization constraint $x=z$ is expressed in terms of hard and soft constraints by the terms $\lambda^{T}(x-z)$ and $1 / 2 \gamma\|x-z\|_{2}^{2}$ respectively.

The corresponding Lagrange programming network for (18) and (19) is given by

$$
\left\{\begin{array}{l}
\dot{x}=f(x)-\gamma(x-z)-\lambda \\
\dot{z}=g(z)+\gamma(x-z)+\lambda \\
\dot{\lambda}=x-z
\end{array}\right.
$$


after application of (10) and (17). We modify this further into the following synchronization scheme with mutual coupling

$$
\Sigma_{\left\{x, z, \lambda \mid \gamma_{1}, \gamma_{2}\right\}}^{1}:\left\{\begin{array}{l}
\dot{x}=f(x)-\gamma_{1}(x-z)-\lambda \\
\dot{z}=g(z)+\gamma_{2}(x-z)+\lambda \\
\dot{\lambda}=x-z
\end{array}\right.
$$

which is characterized by two positive real coupling constants $\gamma_{1}, \gamma_{2}$ instead of $\gamma$ in (20). Master-slave synchronization is a special case of (21) by taking one of the constants $\gamma_{1}, \gamma_{2}$ equal to zero. Here $\Sigma_{\{\nu \mid \theta\}}$ denotes a system of differential equations with state vector $\nu$ for given parameters $\theta$. form

Hence, classical synchronization schemes of the

$$
\Sigma_{\left\{x, z \mid \gamma_{1}, \gamma_{2}\right\}}^{2}:\left\{\begin{array}{l}
\dot{x}=f(x)-\gamma_{1}(x-z) \\
\dot{z}=g(z)+\gamma_{2}(x-z)
\end{array}\right.
$$

could be considered as a special case of (21) with soft constraining only, instead of hard together with soft constraints.

As illustrated for example in [Suykens et al., 1999], synchronization can often only be achieved up to a small synchronization error. In this sense the performance of the Lagrange programming network can be enhanced by modifying the problem formulation (18) into:

$$
\begin{aligned}
\min _{x, z \in \mathbb{R}^{n}} J(x, z, \varepsilon)= & U_{1}(x)+U_{2}(z) \\
& +\frac{1}{2} \gamma\|x-z\|_{2}^{2}+\frac{1}{2} c \varepsilon^{T} \varepsilon
\end{aligned}
$$

such that $\quad x-z+\varepsilon=0$

with augmented Lagrangian

$$
\begin{aligned}
\mathcal{L}_{\gamma, c}(x, z, \varepsilon, \lambda)= & U_{1}(x)+U_{2}(z)+\frac{1}{2} \gamma\|x-z\|_{2}^{2} \\
& +\frac{1}{2} c \varepsilon^{T} \varepsilon+\lambda^{T}(x-z+\varepsilon)
\end{aligned}
$$

where $c$ is a positive real constant. This formulation tolerates a synchronization error $\varepsilon$ which is minimized within the cost function $J$.
From (23) and (24) one obtains the Lagrange programming network

$$
\Sigma_{\left\{x, z, \varepsilon, \lambda \mid \gamma_{1}, \gamma_{2}, c\right\}}^{3}:\left\{\begin{array}{l}
\dot{x}=f(x)-\gamma_{1}(x-z)-\lambda \\
\dot{z}=g(z)+\gamma_{2}(x-z)+\lambda \\
\dot{\varepsilon}=-c \varepsilon-\lambda \\
\dot{\lambda}=x-z+\varepsilon
\end{array}\right.
$$

after applying the master-slave heuristic of (21). We have to emphasize that in order to simulate each of the synchronization schemes $\Sigma$ no construction of the underlying potential functions $U_{1}, U_{2}$ has to be made. The schemes are applied with given systems $f, g$.

Furthermore, note that the synchronization constraint in (18) is an algebraic constraint which the Lagrange programming network has to realize. This constraint is only achieved in an asymptotical sense which is basically different from the way similar problems are analyzed in the theory of differential-algebraic equations [Beardmore \& Song, 1998], with applications e.g. to constrained Hamiltonian systems.

\section{Partial and \\ Generalized Synchronization}

In the case of partial synchronization one aims at synchronizing e.g. only one state variable instead of the full state vector. We illustrate how this can be done within the Lagrange programming framework.

Let us define two output variables $p, q \in \mathbb{R}$ with

$$
\left\{\begin{array} { l } 
{ \dot { x } = f ( x ) } \\
{ p = w ^ { T } x }
\end{array} \quad \left\{\begin{array}{l}
\dot{z}=g(z) \\
q=w^{T} z .
\end{array}\right.\right.
$$

Similar to (24), defining the Lagrangian

$$
\begin{aligned}
\mathcal{L}_{\gamma, c}(x, z, \varepsilon, \lambda)= & U_{1}(x)+U_{2}(z)+\frac{1}{2} \gamma(p-q)^{2} \\
& +\frac{1}{2} c \varepsilon^{2}+\lambda(p-q+\varepsilon)
\end{aligned}
$$

for (26) yields the Lagrange programming network

$$
\Sigma_{\left\{x, z, \varepsilon, \lambda \mid \gamma_{1}, \gamma_{2}, c\right\}}^{4}:\left\{\begin{array}{l}
\dot{x}=f(x)-\gamma_{1}(p-q) w-\lambda w \\
\dot{z}=g(z)+\gamma_{2}(p-q) w+\lambda w \\
\dot{\varepsilon}=-c \varepsilon-\lambda \\
\dot{\lambda}=p-q+\varepsilon
\end{array}\right.
$$


where $\varepsilon(t), \lambda(t) \in \mathbb{R}$ and $\gamma$ has been split into the constants $\gamma_{1}, \gamma_{2}$. This network aims at achieving partial synchronization $|p-q| \rightarrow 0$ as $t \rightarrow \infty$.

Finally, generalized synchronization can be expressed by the optimization problem

$$
\begin{aligned}
& \min _{x, z \in \mathbb{R}^{n}, \varepsilon \in \mathbb{R}^{m}} J(x, z, \varepsilon) \\
& =U_{1}(x)+U_{2}(z)+\frac{1}{2} \gamma \psi(x, z)^{T} \psi(x, z)+\frac{1}{2} c \varepsilon^{T} \varepsilon
\end{aligned}
$$

such that

$$
\psi(x, z)+\varepsilon=0
$$

where $\psi(\cdot, \cdot): \mathbb{R}^{n} \times \mathbb{R}^{n} \rightarrow \mathbb{R}^{m}$ characterizes the synchronization manifold that has to be reached. From the Lagrangian

$$
\begin{aligned}
\mathcal{L}_{\gamma, c}(x, z, \varepsilon, \lambda) & \\
= & U_{1}(x)+U_{2}(z)+\frac{1}{2} \gamma \psi(x, z)^{T} \psi(x, z) \\
& +\frac{1}{2} c \varepsilon^{T} \varepsilon+\lambda^{T}[\psi(x, z)+\varepsilon]
\end{aligned}
$$

one obtains the Lagrange programming network

$$
\Sigma_{\left\{x, z, \varepsilon, \lambda \mid \gamma_{1}, \gamma_{2}, c\right\}}^{5}:\left\{\begin{aligned}
\dot{x}= & f(x)-\gamma_{1}\left(\frac{\partial \psi}{\partial x}\right)^{T} \psi \\
& -\left(\frac{\partial \psi}{\partial x}\right)^{T} \lambda \\
\dot{z}= & g(z)-\gamma_{2}\left(\frac{\partial \psi}{\partial z}\right)^{T} \psi \\
& -\left(\frac{\partial \psi}{\partial z}\right)^{T} \lambda \\
\dot{\varepsilon}= & -c \varepsilon-\lambda \\
\dot{\lambda} & =\psi(x, z)+\varepsilon
\end{aligned}\right.
$$

after applying the master-slave heuristic.

\section{Simulation Examples}

In this section we present simulation examples of the Lagrange programming networks $\Sigma^{1}, \Sigma^{2}, \ldots, \Sigma^{5}$ by taking Chua's circuit, Lorenz attractor and $n$-scroll circuits as examples for $f, g$. Chua's circuit is given by the equations

$$
\left\{\begin{array}{l}
\dot{x}_{1}=a\left[x_{2}-\phi\left(x_{1}\right)\right] \\
\dot{x}_{2}=x_{1}-x_{2}+x_{3} \\
\dot{x}_{3}=-b x_{2}
\end{array}\right.
$$

with nonlinear characteristic

$$
\phi\left(x_{1}\right)=m_{1} x_{1}+\frac{1}{2}\left(m_{0}-m_{1}\right)\left(\left|x_{1}+1\right|-\left|x_{1}-1\right|\right)
$$

and parameters $a=9, b=14.286, m_{0}=-1 / 7$, $m_{1}=2 / 7$, in order to obtain the double-scroll attractor [Chua et al., 1986; Chua, 1994; Madan, 1993]. For $a=8$ and $a=5$ one obtains the case of periodic and sink behavior respectively. By introducing additional breakpoints in the PWL characteristic (33) one obtains a generalized Chua's circuit for (32) with nonlinearity

$$
\begin{aligned}
\phi\left(x_{1}\right)= & m_{2 q-1} x_{1} \\
& +\frac{1}{2} \sum_{i=1}^{2 q-1}\left(m_{i-1}-m_{i}\right)\left(\left|x_{1}+c_{i}\right|-\left|x_{1}-c_{i}\right|\right)
\end{aligned}
$$

which exhibits $n$-scroll attractors where $n$ denotes a natural number and for $q=1$ one obtains Chua's circuit (32) and (33) [Suykens et al., 1997b; Yalcin et al., 1999]. In order to obtain a 5-scroll attractor one sets $q=3$ with $m=$ $[0.9 / 7 ;-3 / 7 ; 3.5 / 7 ;-2.7 / 7 ; 4 / 7 ;-2.4 / 7]$ and $c=$ $[1 ; 2.15 ; 3.6 ; 6.2 ; 9]$ in $(34)$. The Lorenz system [Lorenz, 1963; Chen \& Dong, 1998] is given by the equations

$$
\left\{\begin{array}{l}
\dot{x}_{1}=\sigma_{l}\left(x_{2}-x_{1}\right) \\
\dot{x}_{2}=-x_{1} x_{3}+r_{l} x_{1}-x_{2} \\
\dot{x}_{3}=x_{1} x_{2}-b_{l} x_{3}
\end{array}\right.
$$

with the Lorenz attractor obtained by $\sigma_{l}=10$, $r_{l}=28, b_{l}=8 / 3$. We apply the synchronization schemes by assuming the existence of a potential function for each of the subsystems, eventually in a more generalized sense than characterized by (17). Strictly speaking, in fact smooth versions to Chua's circuit and $n$-scroll circuits [Huang et al., 1996; Ponomarenko \& Matrosov, 1996] should be considered. However, simulation results suggest that the piecewise-linear nature of the circuits does not pose a problem in the examples that have been investigated.

In Fig. 1 a comparison is made between the standard scheme $\Sigma^{2}$ and the Lagrange programming networks $\Sigma^{1}, \Sigma^{3}$ that implement synchronization as a hard constraint. The example makes 

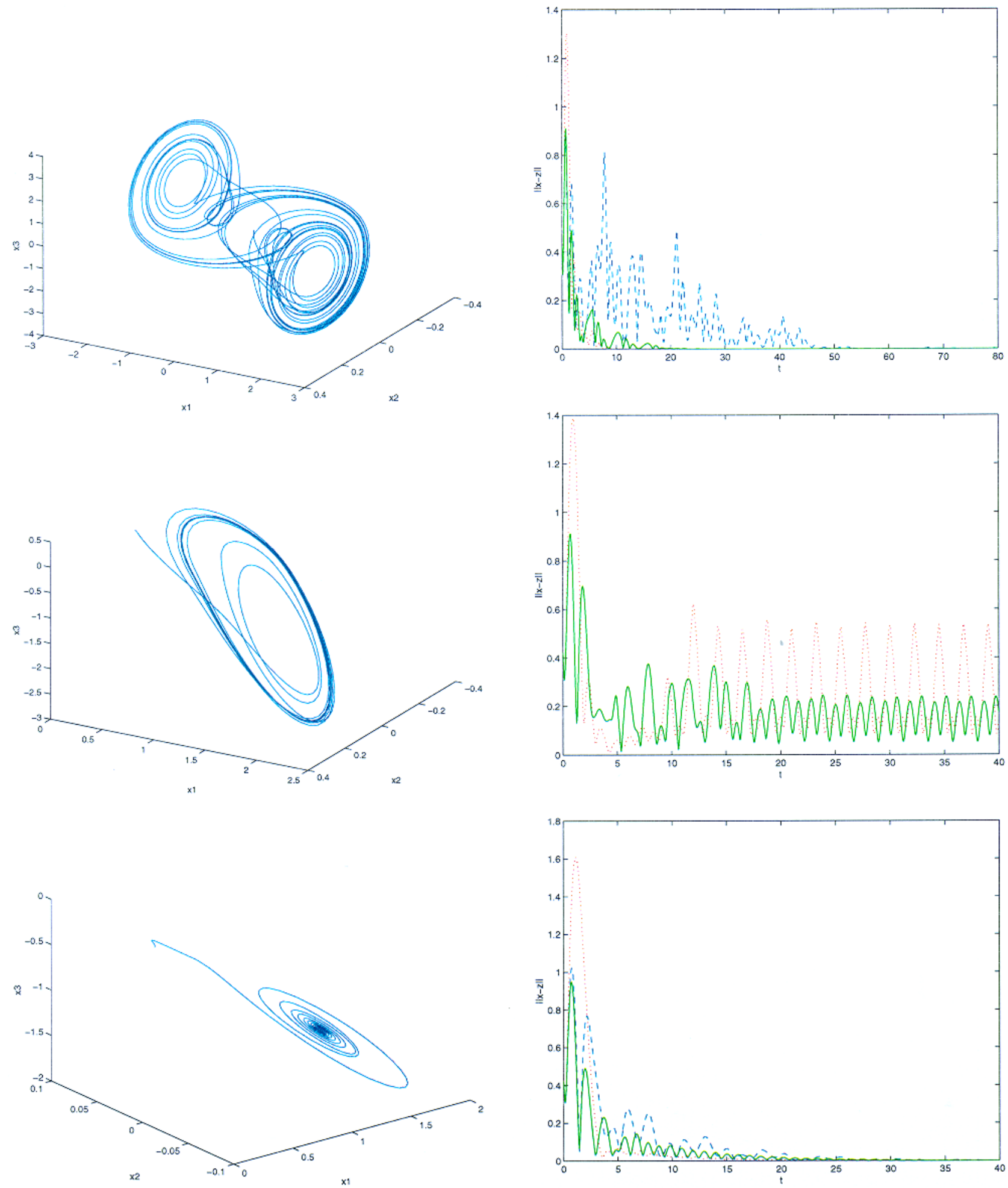

Fig. 1. Comparison of master-slave synchronization schemes with hard and soft constraining applied to Chua's circuits: [Right] $\Sigma_{\{x, z, \lambda \mid 0,1\}}^{1}$ (blue dashed), $\Sigma_{\{x, z \mid 0,1\}}^{2}$ (red dotted), $\Sigma_{\{x, z, \varepsilon, \lambda \mid 0,1,1\}}^{3}$ (green solid) where $\Sigma^{3}$ has the best performance; [Left] in each of the cases $f(x)$ corresponds to a Chua's circuit with $a=9$ (double-scroll) and $g(z)$ to Chua's circuits with $a=9$ (double-scroll) [Top], $a=8$ (periodic) [Middle] and $a=5$ (sink) [Bottom]. In all cases the initial states were chosen as $x(0)=[0.1 ; 0 ;-0.1]$ and $z(0)=[0.2 ; 0.1 ; 0.3]$. 


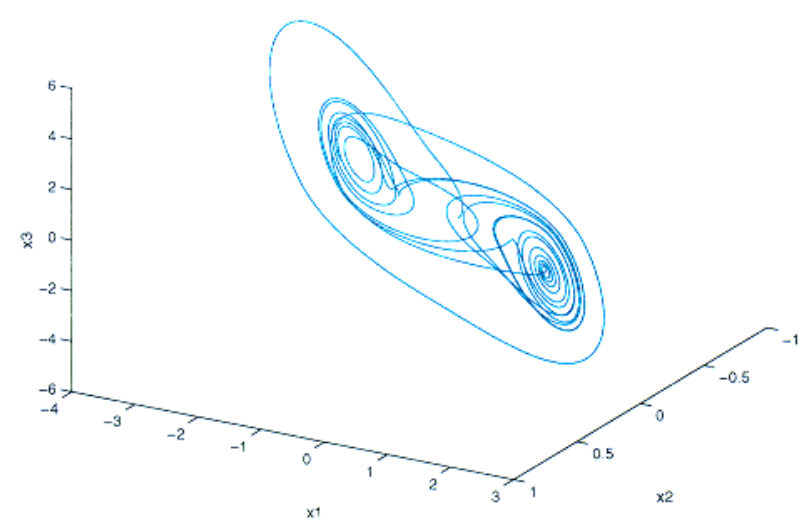

(a)

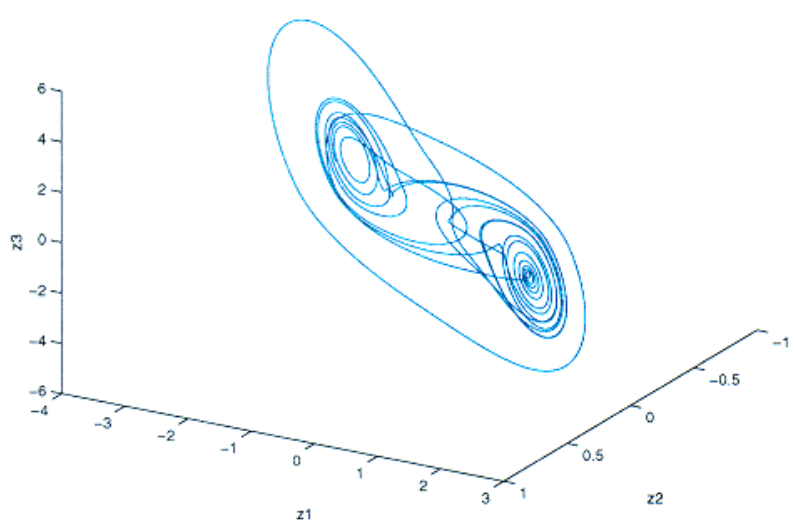

(c)

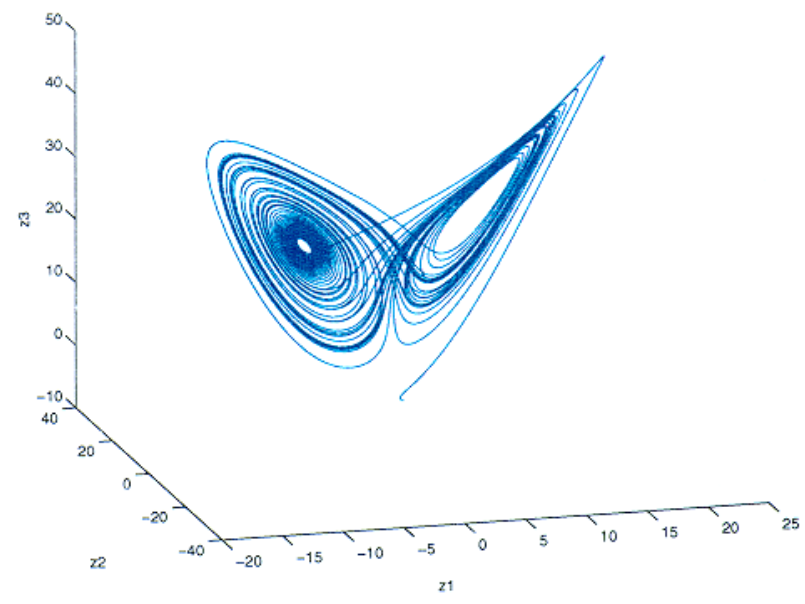

(b)

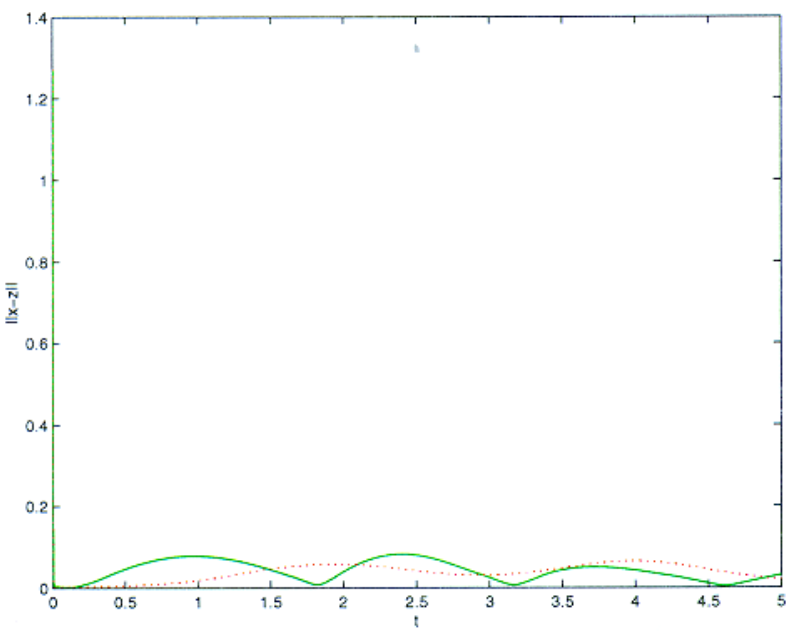

(d)

Fig. 2. Master-slave synchronization by hard and soft constraining applied to Chua's circuit and Lorenz attractor: (a) Chua's circuit with double-scroll as master system $f(x)$; (b) Lorenz attractor as uncontrolled slave system $g(z)$; (c) Lorenz attractor controlled to a double-scroll by synchronization. In these simulations the Lagrange programming network $\Sigma_{\{x, z, \varepsilon, \lambda \mid 0,1000,1\}}^{3}$ has been applied; (d) Comparison between $\Sigma_{\{x, z, \varepsilon, \lambda \mid 0,1000,1\}}^{3}$ and $\Sigma_{\{x, z \mid 0,1000\}}^{2}$. The performance is comparable due to the large value of $\gamma_{2}$. In all simulations the initial states were chosen as $x(0)=[0.1 ; 0 ;-0.1]$ and $z(0)=[1 ; 0 ;-1]$. 


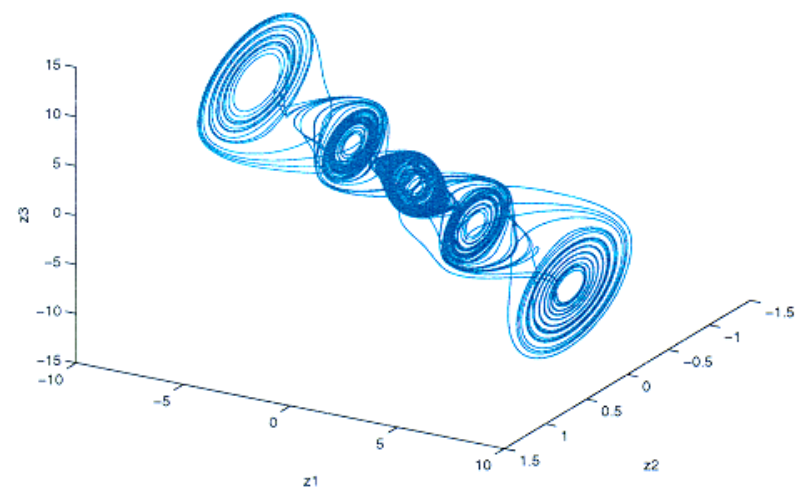

(a)

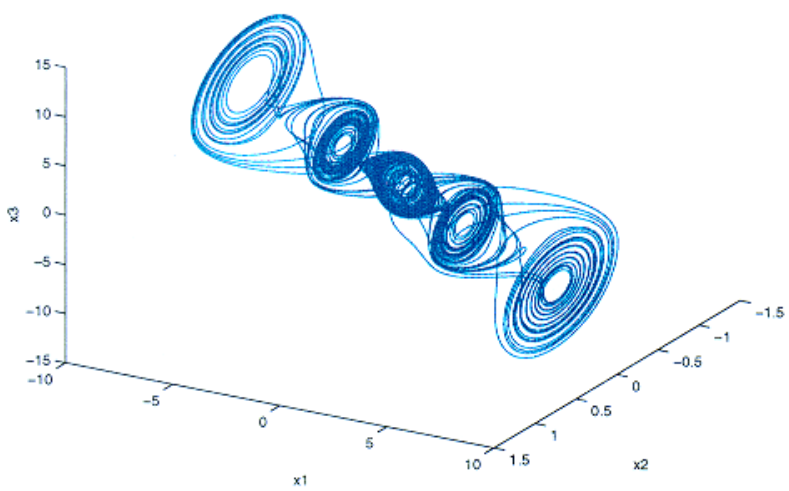

(c)

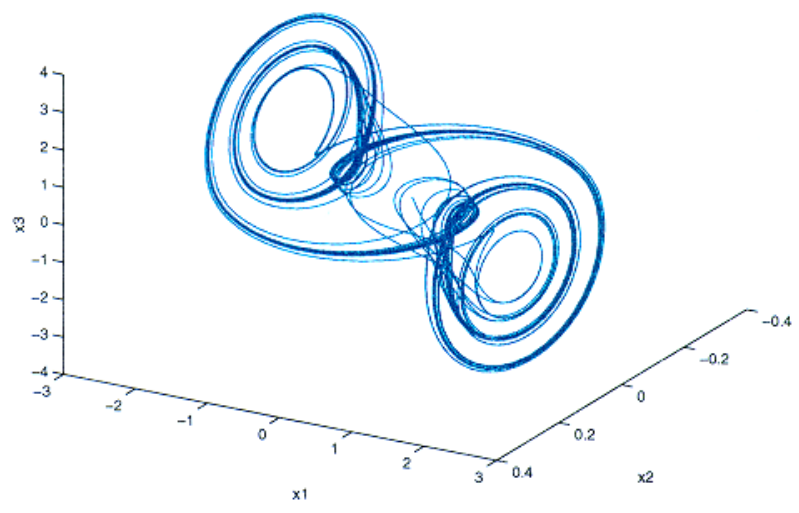

(b)

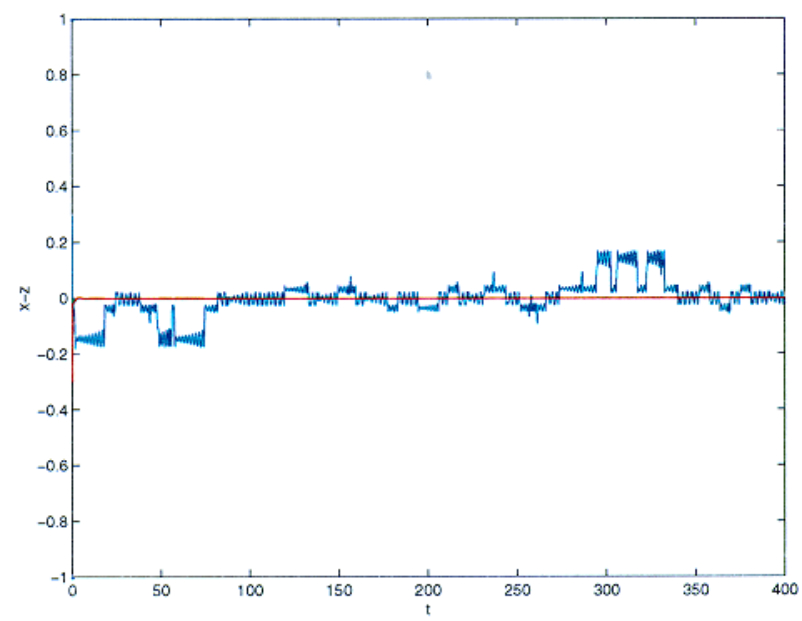

(d)

Fig. 3. Master-slave synchronization by hard and soft constraining applied to 5-scroll circuit and Chua's circuit: (a) 5scroll circuit as master system $f(x)$; (b) Chua's circuit with double-scroll as uncontrolled slave system $g(z)$; (c) double-scroll controlled to a 5 -scroll by synchronization. In these simulations the Lagrange programming network $\Sigma_{\{x, z, \varepsilon, \lambda \mid 0,100,1\}}^{3}$ has been applied; (d) $x(t)-z(t)$ for the synchronized system. Synchronization is achieved up to a small synchronization error. As initial states were taken $x(0)=[-0.2 ; 0 ; 0.2]$ and $z(0)=[0.1 ; 0 ;-0.1]$. 


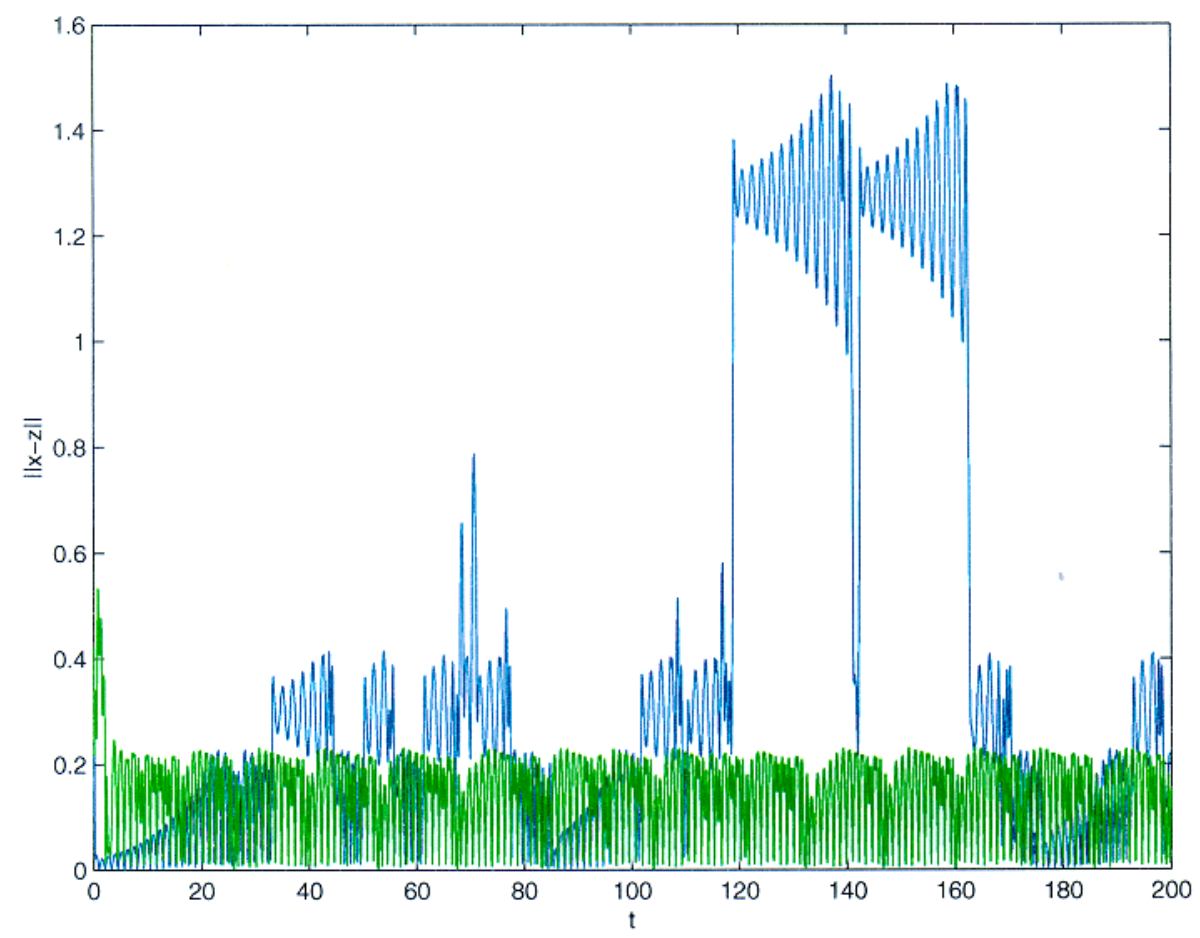

Fig. 4. (Continued) Master-slave synchronization by hard and soft constraining applied to 5-scroll circuit and Chua's circuit: comparison between $\Sigma_{\{x, z, \varepsilon, \lambda \mid 0,10,1\}}^{3}$ and $\Sigma_{\{x, z \mid 0,10,1\}}^{2}$ which shows that a better performance in terms of synchronization error is obtained by applying Lagrange programming networks $\Sigma^{3}$.

use of Chua's circuits with a double-scroll as master system and a double-scroll, periodic behavior and sinks as the slave system. The Lagrange programming network $\Sigma^{3}$ shows the best performance. The synchronization scheme $\Sigma^{3}$ that has been simulated for this example of two Chua's circuits is

$$
\left\{\begin{array}{l}
\dot{x}_{1}=a\left[x_{2}-\phi\left(x_{1}\right)\right]-\gamma_{1}\left(x_{1}-z_{1}\right)-\lambda_{1} \\
\dot{x}_{2}=x_{1}-x_{2}+x_{3}-\gamma_{1}\left(x_{2}-z_{2}\right)-\lambda_{2} \\
\dot{x}_{3}=-b x_{2}-\gamma_{1}\left(x_{3}-z_{3}\right)-\lambda_{3} \\
\dot{z}_{1}=a\left[z_{2}-\phi\left(z_{1}\right)\right]+\gamma_{2}\left(x_{1}-z_{1}\right)+\lambda_{1} \\
\dot{z}_{2}=z_{1}-z_{2}+z_{3}+\gamma_{2}\left(x_{2}-z_{2}\right)+\lambda_{2} \\
\dot{z}_{3}=-b z_{2}+\gamma_{2}\left(x_{3}-z_{3}\right)+\lambda_{3} \\
\dot{\varepsilon}_{1}=-c \varepsilon_{1}-\lambda_{1} \\
\dot{\varepsilon}_{2}=-c \varepsilon_{2}-\lambda_{2} \\
\dot{\varepsilon}_{3}=-c \varepsilon_{3}-\lambda_{3} \\
\dot{\lambda}_{1}=x_{1}-z_{1}+\varepsilon_{1} \\
\dot{\lambda}_{2}=x_{2}-z_{2}+\varepsilon_{2} \\
\dot{\lambda}_{3}=x_{3}-z_{3}+\varepsilon_{3} .
\end{array}\right.
$$

In Fig. 2 master-slave synchronization with hard and soft constraining between Chua's circuit and Lorenz attractor is shown using $\Sigma^{3}$. When the value of $\gamma_{2}$ is taken as large $\left(\gamma_{1}=0\right.$ for master-slave synchronization) the performance of $\Sigma^{2}$ and $\Sigma^{3}$ is comparable in this example. In Fig. 3 masterslave synchronization between a 5-scroll attractor and the double-scroll is shown by applying $\Sigma^{3}$ for a smaller value of $\gamma_{2}$. Synchronization is achieved up to a small synchronization error. Figure 4 shows the benefit of applying Lagrange programming networks $\Sigma^{3}$ which results in smaller synchronization errors compared to $\Sigma^{1}$. In Fig. 5 partial synchronization is illustrated on identical and nonidentical Chua's circuits by applying $\Sigma^{4}$. For larger values of $\gamma_{2}$ in the master-slave scheme the complete state vector becomes synchronized, while for smaller values only $x_{2}, z_{2}$ synchronize when $w=[0 ; 1 ; 0]$. Figures 6 and 7 show generalized synchronization using Lagrange programming networks $\Sigma^{5}$ for identical and nonidentical Chua's circuits. The target synchronization manifold here is characterized by $\psi(x, z)=(x-z)^{T}(x-z)-1=0$. This manifold is reached up to a small synchronization error. 

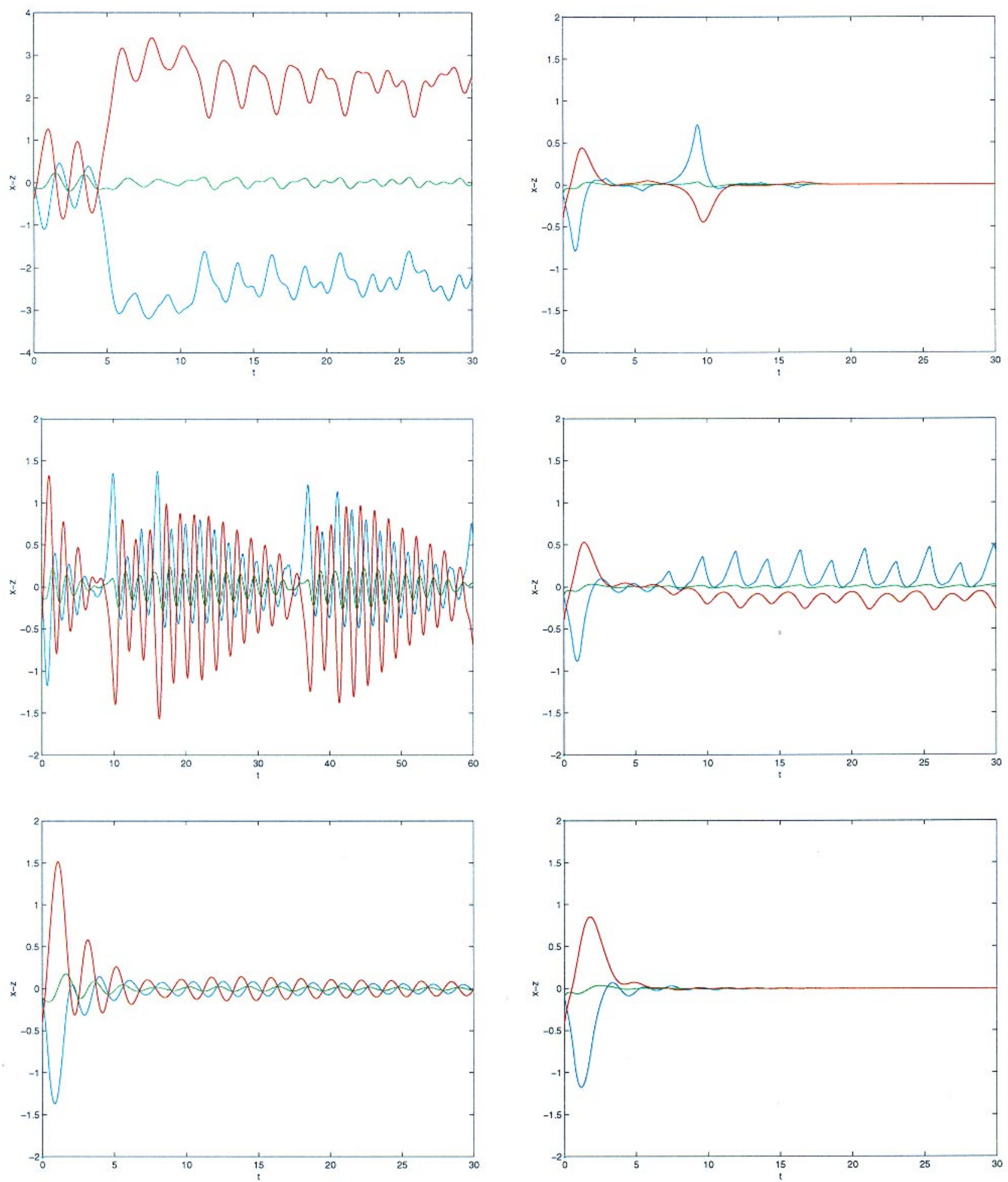

Fig. 5. Partial synchronization using Lagrange programming networks $\Sigma_{\{x, z, \varepsilon, \lambda \mid 0,1,1\}}^{4}$ (Left) and $\Sigma_{\{x, z, \varepsilon, \lambda \mid 0,10,1\}}^{4}$ (Right) illustrated on Chua's circuits. In all cases a double-scroll attractor is chosen for $f(x)$. [Top] double scroll $(a=9)$ as slave system $g(z)$; [Middle] Chua's circuit with $a=8$ (periodic) as slave system $g(z)$; [Bottom] Chua's circuit with $a=5$ (sink) as slave system $g(z)$. In the simulations $w=[0 ; 1 ; 0]$ has been taken. This leads to partial synchronization of $x_{2}-z_{2}$ (green). For large values of $\gamma_{2}$ all state variables become synchronized. 


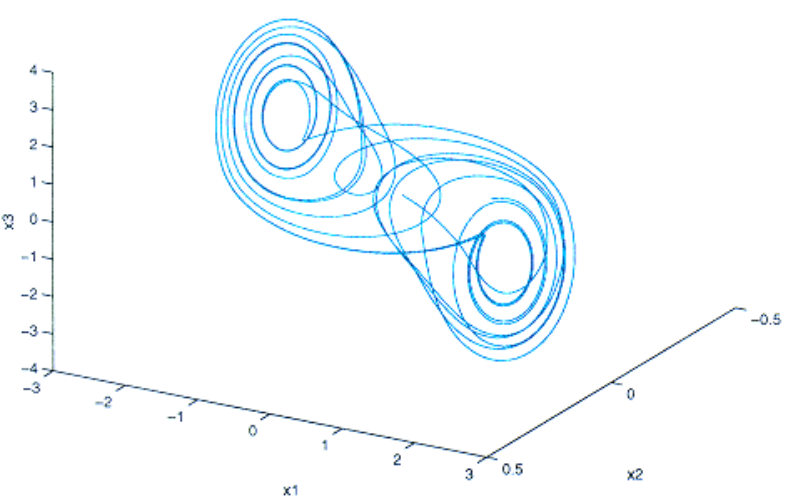

(a)

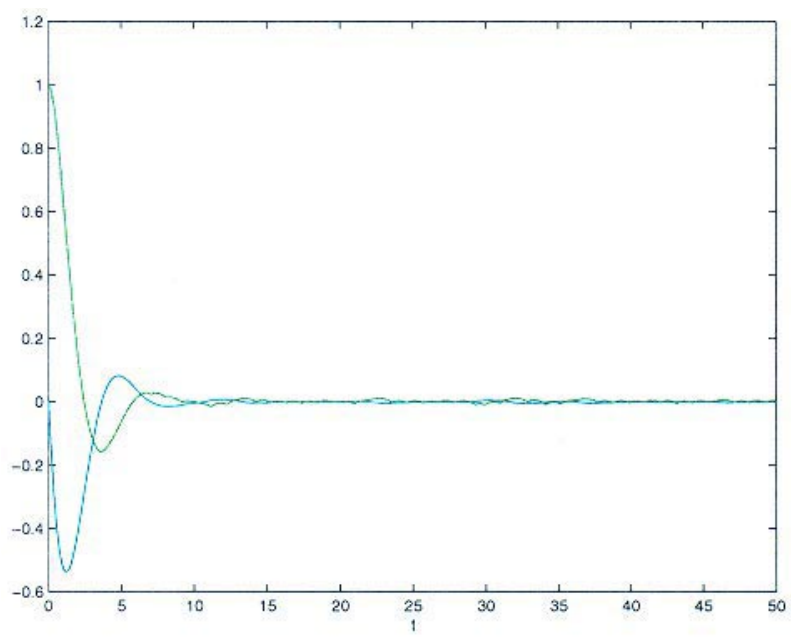

(c)

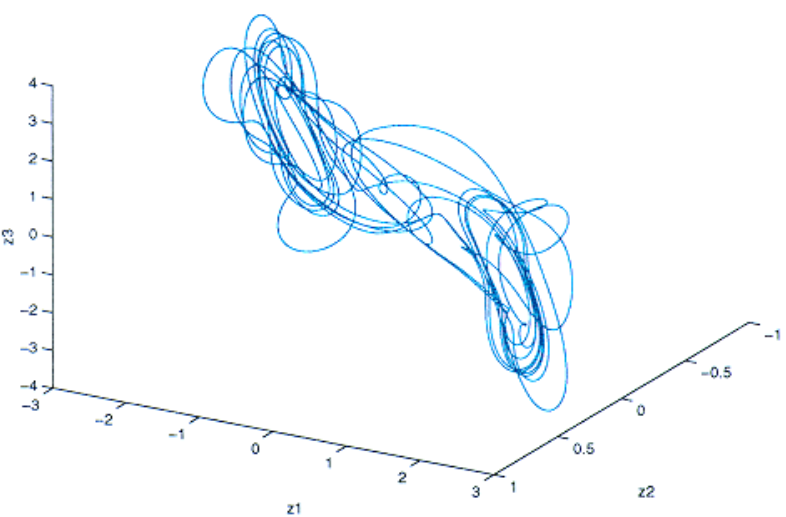

(b)

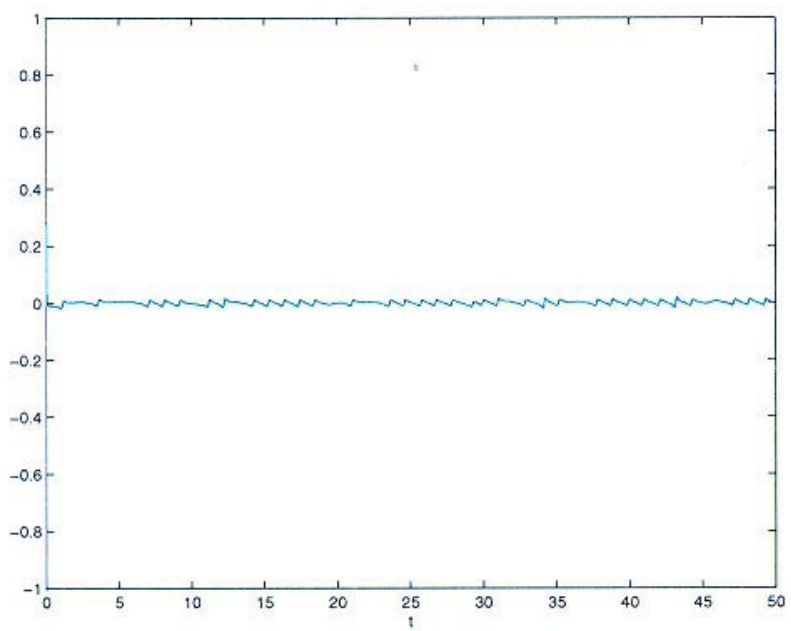

(d)

Fig. 6. Generalized synchronization using Lagrange programming networks $\Sigma_{\{x, z, \varepsilon, \lambda \mid 0,100,1\}}^{5}$ illustrated on identical Chua's circuits with double-scroll attractors for $f(x)$ and $g(z)$. The target synchronization manifold is characterized by $\psi(x, z)=$ $(x-z)^{T}(x-z)-1=0$. (a) Chua's circuit with double-scroll as master system $f(x)$; (b) controlled double-scroll at slave system after synchronization in the generalized sense; (c) $\varepsilon(t), \lambda(t) ;(\mathrm{d})\|\psi(x(t), z(t))\|_{2}$ showing that the synchronization manifold is reached up to a small synchronization error. 

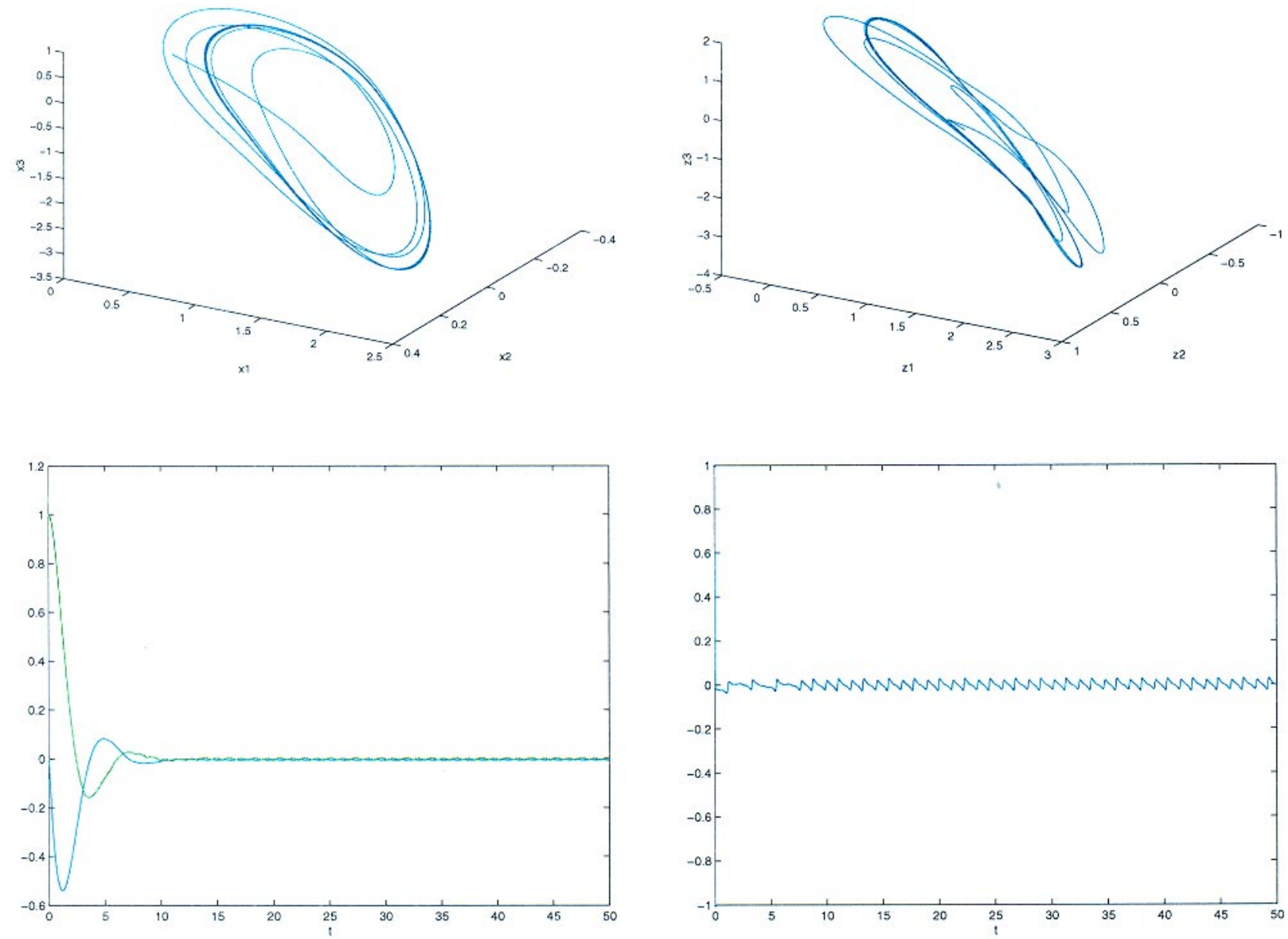

Fig. 7. (Continued) Similar results as in Fig. 6 but with double-scroll master system as $f(x)$ and Chua's circuit with $a=8$ (periodic) as $g(z)$ slave system.

\section{Conclusions}

We introduced methods of Lagrange programming networks for chaos synchronization. The main difference with other synchronization methods is that in Lagrange programming network approach synchronization is implemented both as a hard and soft constraint. Classical schemes may be regarded as soft constraining only. We have shown on simulation examples of full state synchronization, partial and generalized synchronization how the performance can be improved by applying Lagrange programming networks. This has been shown for identical as well as nonidentical chaotic systems with Chua's circuit, Lorenz system and $n$-scroll circuits as examples. From the investigated Lagrange programming networks, the version which takes into account an explicit formulation of the synchronization error yields the best performance. All this is based upon a new interpretation of synchronization problems within a Lagrangian framework. The contribution of this paper is mainly conceptual in the sense of establishing new links between synchronization, neural networks and constrained optimization. Although the results of this paper are supported by many simulation results, a better understanding of the existence of potential functions in the generalized sense of potential function theory is needed within this context.

\section{Acknowledgments}

This research work was carried out at the ESAT laboratory and the Interdisciplinary Center of Neural Networks ICNN of the Katholieke Universiteit Leuven, in the framework of the Belgian Programme on Interuniversity Poles of Attraction, initiated by the Belgian State, Prime Minister's Office for Science, Technology and Culture (IUAP P4-02) and in 
the framework of a Concerted Action Project MIPS (Modelbased Information Processing Systems) of the Flemish Community. J. Suykens is a postdoctoral researcher with the National Fund for Scientific Research FWO-Flanders.

\section{References}

Beardmore, R. E. \& Song, Y. H. [1998] "Differentialalgebraic equations: A tutorial review," Int. J. Bifurcation and Chaos 8(7), 1399-1411.

Chen, G. \& Dong, X. [1998] From Chaos to Order Perspectives, Methodologies, and Applications (World Scientific, Singapore).

Chua, L. O. [1994] "Chua's circuit 10 years later," Int. J. Circuit Th. Appl. 22, 279-305.

Chua, L. O., Komuro, M. \& Matsumoto, T. [1986] "The double scroll family," IEEE Trans. Circuits Syst. I 33(11), 1072-1118.

Cichocki, A. \& Unbehauen, R. [1994] Neural Networks for Optimization and Signal Processing (Wiley, Chichester).

Fletcher, R. [1987] Practical Methods of Optimization (John Wiley, Chichester, NY).

Hazewinkel, M. (ed.) [1991] Encyclopaedia of Mathematics (Kluwer Academic Publishers, Boston).

Hopfield, J. J. [1984] "Neurons with graded response have collective properties like those of two-state neurons," Proc. Natl. Acad. Sci. 81, pp. 3088-3092.

Huang, A., Pivka, L., Wu, C.-W. \& Franz, M. [1996] "Chua's equation with cubic nonlinearity," Int. J. Bifurcation and Chaos 6(8), 2175-2222.

Kapitaniak, T. \& Chua, L. O. [1994] "Hyperchaotic attractors of unidirectionally-coupled Chua's circuits," Int. J. Bifurcation and Chaos 4(2), 477-482.

Kapitaniak, T., Chua, L. O. \& Zhong, G.-Q. [1994] "Experimental synchronization of chaos using continuous control," Int. J. Bifurcation and Chaos 4(2), 483-488.

Kennedy, M. P. \& Chua, L. O. [1988] "Neural networks for nonlinear programming," IEEE Trans. Circuits Syst. 35, 554-562.

Kocarev, L. \& Parlitz, U. [1996] "Generalized synchronization, predictability, and equivalence of unidirectionally coupled dynamical systems," Phys. Rev. Lett. 76(11), 1816-1819.
Kocarev, L., Shang, A. \& Chua, L. O. [1993] "Transitions in dynamical regimes by driving: A unified method of control and synchronization of chaos," Int. J. Bifurcation and Chaos 3(2), 479-483.

Lorenz, E. N. [1963] "Deterministic nonperiodic flow," J. Atmos. Sci. 20, 130-141.

Madan, R. N. (Guest Editor) [1993] Chua's Circuit: A Paradigm for Chaos (World Scientific, Singapore).

Morgül, O. \& Solak E. [1997] "On the synchronization of chaotic systems by using state observers," Int. J. Bifurcation and Chaos 7(6), 1307-1322.

Ponomarenko V. P. \& Matrosov V. V. [1996] "Nonlinear dynamics of multistable Chua's circuits," Int. J. Bifurcation and Chaos 6(11), 2087-2096.

Suykens, J. A. K., Curran, P. F. \& Chua, L. O. [1997a] "Master-slave synchronization using dynamic output feedback," Int. J. Bifurcation and Chaos 7(3), 671-679.

Suykens, J. A. K., Huang, A. \& Chua, L. O. [1997b] "A family of $n$-scroll attractors from a generalized Chua's circuit," Archiv für Elektronik und Ubertragungstechnik (Int. J. Electron. Commun.) 51(3), 131-138.

Suykens, J. A. K., Curran, P. F., Vandewalle, J. \& Chua, L. O. [1997c] "Robust nonlinear $\mathrm{H}_{\infty}$ synchronization of chaotic Lur'e systems," IEEE Trans. Circuits Syst. I, Special issue on Chaos Synchronization, Control and Applications 44(10), 891-904.

Suykens, J. A. K., Curran, P. F. \& Chua, L. O. [1999] "Robust synthesis for master-slave synchronization of Lur'e systems," IEEE Trans. Circuits Syst. I 46(7), 841-850.

Weiss L., Mathis W. \& Trajkovic Lj. [1998] "A generalization of Brayton-Moser's mixed potential function," IEEE Trans. Circuits Syst. I 45(4), 423-427.

Wu, C. W. \& Chua, L. O. [1994] "A unified framework for synchronization and control of dynamical systems," Int. J. Bifurcation and Chaos 4(4), 979-989.

Yalcin, M. E., Suykens, J. A. K. \& Vandewalle, J. [2000] "Experimental confirmation of 3- and 5-scroll attractors from a generalized Chua's circuit," IEEE Trans. Circuits Syst. I, to appear.

Zhang, S. \& Constantinides, A. G. [1992] "Lagrange programming neural networks," IEEE Trans. Circuits Syst. II 39(7), 441-452.

Zubov, V. I. [1997] Mathematical Theory of the Motion Stability (Saint-Petersburg, Russia). 